Proceedings

\title{
An Italian Registry on Risk Factors for Venous Thromboembolism in Blood Donors Clinicaltrials. Gov: Nct03282747 †
}

\author{
Elvira Grandone ${ }^{1, *}$, Angelo Ostuni ${ }^{2}$, Lazzaro di Mauro ${ }^{3}$, Nadia Coffetti ${ }^{4}$, Anna Turrini ${ }^{5}$, \\ Giobatta Cavallero ${ }^{6}$, Patrizia Vergura ${ }^{1}$, Filomena Cappucci ${ }^{1}$, Antonio de Laurenzo ${ }^{1}$, \\ Michela Villani ${ }^{1}$, Nicola Di Renzo ${ }^{7}$, Alberto Tosetto ${ }^{8}$ and Maurizio Margaglione ${ }^{9}$ \\ 1 Thrombosis and Haemostasis Centre, Fondazione IRCCS Casa Sollievo della Sofferenza, Viale Cappuccini, \\ 71013 San Giovanni Rotondo, Italy; e.grandone@operapadrepio.it (E.G.); p.vergura@operapadrepio.it (P.V.); \\ f.cappucci@operapadrepio.it (F.C.); antonio.delaurenzo@gmail.com (A.d.L.); \\ m.villani@operapadrepio.it (M.V.) \\ 2 Transfusion Medicine, Azienda Ospedaliero-Universitaria Consorziale, Policlinico di Bari, Piazza Giulio \\ Cesare, 70124 Bari, Italy; angelostuni@gmail.com \\ 3 Clinical Laboratory and Transfusion Medicine, Fondazione IRCCS Casa Sollievo della Sofferenza, Viale \\ Cappuccini, 71013 San Giovanni Rotondo, Italy; 1.dimauro@operapadrepio.it \\ 4 Immunology and Transfusion Medicine, Azienda Ospedaliera Bolognini, ASST Bergamo Est, via Marconi, \\ 38, 24068 Seriate, Italy; nadia.coffetti@gmail.com \\ 5 Clinical Laboratory and Transfusion Medicine, Ospedale “Sacro Cuore-Don Calabria”, via don A. \\ Sempreboni, 5, 37024 Negrar, Italy; anna.turrini@sacrocuore.it \\ 6 Internal Medicine, Ospedale "Santa Croce e Carle", via Michele Coppino, 26, 12100 Cuneo, Italy; \\ medicina.interna@ospedale.cuneo.it \\ 7 Haematology, Ospedale Vito Fazzi, Piazzetta Muratore, 73100 Lecce, Italy; ematologia.polecce@ausl.le.it \\ 8 Haematology Unit, Ospedale S. Bortolo, via Ferdinando Rodolfi, 37, 36100 Vicenza, Italy; \\ alberto.tosetto@ulssvicenza.it \\ 9 Medical Genetics, University of Foggia, viale Pinto, 71100 Foggia, Italy; maurizio.margaglione@unifg.it \\ * Correspondence: e.grandone@operapadrepio.it; Tel.: +39-0882-416-286 \\ + Presented at the 25th Biennial International Congress on Thrombosis, Venice, Italy, 23-26 May 2018. \\ Published: 25 July 2018
}

Abstract: A registry-based study to evaluate prevalence of common risk factors for vein thrombosis among healthy subjects.

Keywords: vein thrombosis; risk; blood donors

\section{Background}

The impact of several risk factors in the occurrence of venous thrombosis with/without pulmonary embolism, collectively called venous thromboembolism (VTE), is well known in patients who previously suffered from VTE [1]. Little is known about their impact in healthy population.

\section{Materials and Methods}

We evaluated the incidence of risk factors for VTE in a population of Italian blood donors: BMI, blood group, previous transfusions, plaster cast, previous surgery, gross veins, history of venous thrombosis and/or use of anticoagulation drugs. The study started in 1 June 2017. Information were collected by means of a self-administered questionnaire. All analyses were performed using SPSS version 11.0. Odds ratios (ORs) for risk factors for VTE were calculated. Proportions were compared using Fisher exact test or c2-test where appropriate. Adjusted OR and 95\% confidence interval (CI) 
were calculated using logistic regression models that controlled for potential confounding variables such as age, BMI, blood group, surgery, plaster cast, immobilization, transfusion.

\section{Results}

Until November 20th, 5506 questionnaires were collected. 4120 (75.3\%) men and 1354 (24.7\%) women were consecutively enrolled. Mean age (+SD) was $42.7+12.3 \mathrm{yrs}$ in men, $38.4+13.4 \mathrm{yrs}$ in women $(p<0.001)$, BMI was $26.05+4.14$ in men and $24.5+4.93$ in women $(p<0.001)$. Group 0 was observed in $48 \%$ and non- 0 in $52 \%$. With regards to smoking habits, no significant difference was observed between men and women with the exception for ex-smokers (143/1354 women vs. 786/4120 men, $p<0.001)$. A history of vein thrombosis (likely superficial thromboses) was referred by $36(0.7 \%)$ subjects, gross veins by 320/5442 (5.9\%), previous surgery by 1896/5478 (34.6\%). Previous transfusion was reported by 73/5019 (1.5\%) individuals and 236/5268 (4.5\%) had used at least once anticoagulation drugs. At univariate analysis, gross veins, bed rest/plaster cast, surgery and transfusions were associated with vein thrombosis. At logistic regression (see Table 1), a significantly and independent association was found between VTE and gross veins (OR: 15.8, 95\%CI 7.7-32.6), plaster cast/bed rest (OR: 2.3, 95\% CI 1.0-5.3) and transfusion (OR: 5.1, 95\% CI 1.3-19.5).

\section{Discussion}

To the best of our knowledge, this is the first study in a large series of blood donors aimed at investigating the distribution of risk factors for VTE. We find that gross veins, plaster cast/bed rest and previous transfusions are independent risk factors for VTE. Further studies that can confirm and extend these findings are warranted.

Bullet points:

- Assessment of risk factors for VTE is mostly limited to VTE-diagnosed subjects

- Information on risk factors for VTE in healthy subjects are lacking

- Transfusions could confer the risk for VTE

Table 1. Logistic regression.

\begin{tabular}{ccc}
\hline Variables & OR & $\mathbf{9 5 \% ~ C I ~}$ \\
\hline Gross veins & 15.8 & $7.7-32.6$ \\
Plaster cast/Bed rest & 2.3 & $1.0-5.3$ \\
Transfusions & 5.1 & $1.3-19.5$ \\
\hline
\end{tabular}

Conflicts of Interest: The authors declare no conflict of interest.

\section{References}

1. Seeley, R.R.; Stephens, T.D.; Tate, P. Anatomy and Physiology, 4th ed.; The McGraw Hill Companies Inc.: New York, NY, USA, 1998; p. 1098.

(C) 2018 by the authors. Licensee MDPI, Basel, Switzerland. This article is an open access article distributed under the terms and conditions of the Creative Commons Attribution (CC BY) license (http://creativecommons.org/licenses/by/4.0/). 